\title{
A Comprehensive Study of the Thermal and Magnetic Properties of Two-Harmonically Interacting Electrons Confined in a Parabolic GaAs Quantum Dot in a Magnetic Field
}

\author{
A. A. Shukri ${ }^{1} \&$ F. S. Nammas ${ }^{1}$ \\ ${ }^{1}$ Applied Physics Department, Faculty of Science, Tafila Technical University, Tafila, Jordan \\ Correspondence: F. S. Nammas, Applied Physics Department, Faculty of Science, Tafila Technical University, \\ Tafila 66110, Jordan. E-mail: fnammas@ttu.edu.jo
}

Received: April 12, 2019

Accepted: May 5, 2019

Online Published: May 31, 2019

doi:10.5539/apr.v11n3p76

URL: http://dx.doi.org/10.5539/apr.v11n3p76

\begin{abstract}
The thermal and magnetic properties of a parabolic GaAs quantum dot for two-Harmonically interacting electrons when it exposed to an external magnetic field, taking into account the spin-Zeeman energy are investigated using the canonical ensemble approach. The effect of spin on these properties is also investigated. With the possibility of a basic and physically sensible model of electron-electron interaction, the issue is precisely soluble. We found a Schottky-like anomaly in the heat capacity at low temperature, while it saturates to the $4 \mathrm{k}_{\mathrm{B}}$ value as the temperature increases. Also it is noted that entropy enhances with temperature as expected. However as a function of a magnetic field, a peak structure is observed in heat capacity at very low values of magnetic field, while it saturates to the $2 \mathrm{k}_{\mathrm{B}}$ value as magnetic field increases. Also we noticed that these peaks are not presented in the spinless case. Moreover magnetic field does not show a significant effect on the entropy at high temperatures, but at relatively lower temperatures, the entropy shows a monotonic increase with magnetic field. As a function of the Lande $\mathrm{g}^{*}$ factor, we found a local minima and a double peak-structure in the susceptibility and in the heat capacity at $\mathrm{g}^{*}=0$. It is demonstrated that the favored state for both magnetization and susceptibility is the diamagnetic state. The significant effect of the spin on the magnetic properties of quantum dot is seen at low values of temperature and magnetic field. Moreover, our results showed a very good agreement with reported previous works.
\end{abstract}

Keywords: Quantum Dot, Harmonic E-E Interaction, Heat Capacity, Entropy, Susceptibility, Magnetization

\section{Introduction}

For more than two decades the research area of quantum dots has attracted great attention (Ashoori, 1996; Johnson, 1995; Kastner, 1992; Woggon, 1997; Jacak, Hawrylack, \& Wojs, 1998; Bimberg, Grundmann, \& Ledentsov, 1998; Mukhopadhyay \& Chatterjee, 2001; Kouwenhoven, Austing, \& Tarucha, 2001; Haddad, Nammas, Al Shorman, \& Shukri, 2017). The reason is fundamentally two-fold. First and foremost, the structure of the quantum dots (QDs) exhibits an exciting quantum effect due to their nanoscale extension, in addition their fabrication flexibility enable them to be used extensively in a wide range of potential applications in micro-electronic devices such as solar cells, quantum lasers, quantum computers and single electron transistor. Secondly, QDs are an ideal quantum system that contains a discrete and variable numbers of electrons, therefore QDs are called artificial atoms, so they can be used to examine the predictions in quantum mechanics in a comprehensive and moderated manner.

The confinement potential is the most important feature of a QD. The generalized Kohn theorem together with magneto-optical experiments (Sikorski \& Merkt, 1989; Karrai, Drew, Lee, \& Shayengan, 1989) have proposed that the confinement potential in a QD is less or pretty much parabolic (Peeters, 1990; Yip, 1991; Li, Karrai, Yip, Sarma, \& Drew, 1991). This has leads to a hot pace action in the field of QD's (Maksym \& Chakraborty, 1990; Gu \& Guo, 1993; Johnson \& Payne, 1992; Mukhopadhyay \& Chatterjee, 1996; Mukhopadhyay \& Chatterjee, 1997). It has additionally understood that electron-electron interaction (e-e interaction) guides and controls the properties and the composition of QDs. Unfortunately, multi-electrons QD problem with the e-e interaction does not recognize exactly theoretical solution. Thus, many analytical methods and numerical approximations have been adopted to explore and study a multi-electrons QD problem (Beenakker, 1991; Oaknin, Palacios, Brey, \& 
Tejedor, 1994; Johnson \& Reina, 1992; Reusch \& Grabert, 2003; Emperador, Lipparini, \& Serra, 2006; McEuen, Foxman, Kinaret, Meirav, Kastner, Wingreen, \& Wind, 1992; Harju, Sverdlov, Nieminen, \& Halonen, 1999; Ferconi \& Vignale, 1994; Cipriani, Rosa-Clot, \& Taddei, 2000; Mukhopadhyay \& Chatterjee, 2000; Harju, Sverdlov, Barbiellini, \& Nieminen, 1998; Mukhopadhyay \& Chatterjee, 1999a, 1999b; Liu et al., 2017). While a portion of these techniques are excessively shortsighted, making it impossible to catch the fundamental features of QDs, some are limited just to the ground energy levels, some ignore to incorporate the correlation and exchange effects legitimately, some are unable to manage and work with high electron density and large magnetic field.

Several numerical studies have been applied to QDs to investigate their thermal and magnetic properties (Nammas, Sandouqa, Ghassib, \& Al-Sugheir, 2011; Kuros \& Okopinska, 2015; Maksym \& Chakraborty, 1990; Shaer, Elsaid, \& Elhasan, 2016; Climente, Planelles, \& Movilla, 2004; Shaer, Elsaid, \& Elhasan, n. d.; Boda \& Chatterjee, 2014; Mukhopadhyay \& Chatterjee, 1999; Merkt, Huser, \& Wagner, 1991; Tavernier, Anisimovas, Peeters, Szafran, Adamowski, \& Bednarek, 2003; Yang, MacDonald, \& Johnson, 1993; Hawrylak, 1993). The diagonalization method was used to explore the effect of e-e interaction on the magnetization and susceptibility of a parabolic GaAs QD with two interacting electrons in the presence of a uniform magnetic field and the spin-Zeeman energy (Boda, Kumar, Sankar, \& Chatterjee, 2016). It was found that a sharp diamagnetic peaks were observed in these magnetic properties due to the singlet-triplet transitions and the number and the position of theses peaks depends crucially both on the interaction strength and confinement strength. The same method was used to investigate the magnetization and susceptibility of a Gaussian GaAs QD of two interacting electrons in the presence of a magnetic field with spin-Zeeman term (Boda \& Chatterjee, 1942). It was concluded that the oscillatory behavior seen in these magnetic properties is due to singlet-triplet transitions in the ground state which caused by the e-e interaction. A variational method was used to study the heat capacity for two interacting electrons confined in a two-dimensional parabolic QD (2DPQD) and subjected to an external magnetic field (Shaer, Elsaid, \& Elhasan, 2016). The main result is that the heat capacity shows a peak structure at low temperature and it saturates in the high temperature region at a specific confining frequency. The magnetization, susceptibility and heat capacity have been calculated numerically for two interacting electrons confined in 2DPQD where the spin interaction has been included in the Hamiltonian (De Groote, Horonos, \& Chaplik, 1992). It was shown that, the heat capacity shows an additional structure and the susceptibility exhibits a sharp peak. Recently, the energy spectrum, the magnetic moment and the susceptibility were calculated for two-electron Gaussian GaAs quantum dot with spin-Zeeman term by numerical diagonalization method (Sharma et al., 2019). They concluded that the magnetic moment and the magnetic susceptibility are shown to have zero-temperature diamagnetic peaks due to the exchange-induced singlet-triplet oscillations.

It was understood that e-e interaction effectively affects the energy spectrum of QDs and in this manner numerous studies have investigated the effect of e-e interaction on the electronic properties of QD. But a large part of these investigations depends mainly on approximation and numerical calculations (Oaknin, Palacios, Brey, \& Tejedor, 1994; Johnson \& Reina, 1992; Reusch \& Grabert, 2003; Emperador, Lipparini, \& Serra, 2006; Hawrylak, 1993; Yang, MacDonald, \& Johnson, 1993). Numerical techniques are generally suffered from convergence issues; consequently it might be valuable to look for a potential model that simulates the Coulomb interaction. One of these models is the Johnson-Payne potential (Johnson \& Payne, 1991) that makes the QD Hamiltonian totally solvent with the goal that all many body effects are incorporated accurately (Nammas, 2018; Al Shorman, Nammas, Haddad, \& Shukri, n. d.). Since this model is completely solvable, we won't need to make any other rough estimation. This model depicts the real system well for a particular set of electronic electrons Separation. Therefore, we expect that the basic characteristics of the actual state will be recognized through our calculations, at least for a certain range of electron- electron separation. Several investigations have been made on a few-electron QD to study their thermal and magnetic properties in the presence of either magnetic field or spin-Zeeman interaction or both. In most previous studies the e-e interaction has been taken as Coulomb interaction. As far as we know no investigation has been made on the temperature (magnetic field) -dependent heat capacity, magnetization and susceptibility in a parabolic QD in the presence of both Harmonic e-e interaction and the spin-Zeeman interaction. The motivation of this study is to make an endeavor toward this path. Furthermore, we shall investigate the behavior of the thermal and magnetic properties when the QD is exposed to a uniform magnetic field. For comparison purposes, we have plotted the spinless case in the same figure for all thermal and magnetic properties.

The paper is arranged as follows. In Section 2, we introduce the mathematical formalism of our system. We analyze and discuss the obtained results for GaAs QD in Section 3. In Section 4, we conclude this study with some particular remarks. 


\section{Mathematical Formalism}

In a $2 \mathrm{D}$ system the electron is restricted in one direction but free to move around in the other two, quantization along the confined direction gives rise to an energy sub-band in addition to the usual energy bands arising due to the 2D periodic core potential. The effect on the conduction electron of the periodic core potential is captured by the effective electron mass (Sze, 1981; Sze, 1985; Chang \& Sze, 1996; Liu, 2012). In the effective mass approximation, the Hamiltonian for two interacting electrons confined in a 2DPQD in a perpendicular magnetic field is given by:

$$
\widehat{H}=\sum_{i=1}^{2}\left(\frac{1}{2 m^{*}}\left(\vec{p}_{i}+e \vec{A}\left(\vec{r}_{i}\right)\right)^{2}+\frac{1}{2} m^{*} \omega^{2} r_{i}^{2}\right)+V\left(\vec{r}_{1}, \vec{r}_{2}\right)+\widehat{H}_{s p i n}
$$

where $\vec{r}_{i}$ is the electron's position vector in a QD, $\vec{p}_{i}$ is the momentum operator of electron, $m^{*}$ is the effective mass of the electron; in order to incorporate the effect of the host semiconductor material, $\omega$ is the confinement frequency of the parabolic potential, $\overrightarrow{\mathrm{A}}$ is the vector potential corresponding to the magnetic field $\overrightarrow{\mathrm{B}}=B \hat{k}$, $V\left(\vec{r}_{i}, \vec{r}_{j}\right)$ represents the electron-electron interaction and the spin-Zeeman Hamiltonian is given by $\widehat{H}_{\text {spin }}=$ $\frac{1}{2} \omega_{c} g^{*} \hat{S}_{t o t, z}$, where $g^{*}=-0.44$ is the effective Lande-g factor for GaAs QD, $\omega_{c}=e B / m^{*}$ is the electron's cyclotron frequency, $\hat{S}_{t o t, z}$ is the total z-component of the spin operator.

It might be valuable to search for a model potential which emulates the coulomb interaction potential and makes the Hamiltonian precisely solvable so all the many-body effects are incorporated legitimately. One of these possibilities is the Johnson-Payne potential model (Johnson \& Payne, 1991) and is given by the model potential

$$
V\left(\vec{r}_{1}, \vec{r}_{2}\right)=2 V_{0}-\frac{1}{2} m^{*} \Omega^{2}\left|\vec{r}_{1}-\vec{r}_{2}\right|^{2}
$$

Where $\vec{r}_{1}$ and $\vec{r}_{2}$ are the corresponding position vector of two electron system, $V_{0}$ and $\Omega$ are certain positive parameters which can be fit distinctive sorts of QD. If we choose the gauge of $\vec{A}$ as $\vec{A}=B\left(-\frac{y}{2}, \frac{x}{2}, 0\right)$ then $\vec{A}$ is divergence-less (In quantum mechanics, all computed observable ought to moreover be invariant to such changes. a gauge transformation changes the Hamiltonian and wave function in a way that leaves every recognizable property unaffected, supporting our utilization of the Coulomb gauge in our development of the Hamiltonian operator (Jackson, 1962)). The spin-Zeeman term is included in the Hamiltonian, and then we can write Eq. (1) as

$$
\widehat{H}=\sum_{i=1}^{2}\left(\frac{p_{i}^{2}}{2 m^{*}}+\frac{1}{2} m^{*} \omega_{0}^{2} r_{i}^{2}\right)+2 V_{0}-\frac{1}{2} m^{*} \Omega^{2}\left|\vec{r}_{1}-\vec{r}_{2}\right|^{2}+\frac{1}{2} \omega_{c} \widehat{L}_{t o t, z}+\frac{1}{2} \omega_{c} g^{*} \hat{S}_{t o t, z}
$$

where $\omega_{0}=\sqrt{\omega^{2}+\omega_{c}^{2} / 4}$ is the effective frequency, $\hat{L}_{t o t, z}$ is the total orbital angular momentum along the $\mathrm{z}$ direction. If we introduce center-of-mass coordinates $\overrightarrow{\mathrm{R}}=\left(\vec{r}_{1}+\vec{r}_{2}\right) / \sqrt{2}$ with linear momenta $\overrightarrow{\mathrm{P}}_{R}=$ $\left(\vec{p}_{1}-\vec{p}_{2}\right) / \sqrt{2}$, and relative coordinates $\overrightarrow{\mathrm{r}}=\left(\vec{r}_{1}-\vec{r}_{2}\right) / \sqrt{2}$ with linear momenta $\overrightarrow{\mathrm{P}}_{r}=\left(\vec{p}_{1}+\vec{p}_{2}\right) / \sqrt{2}$, then Schrödinger equation is separable in the new coordinates into three parts, and it can be written as

$$
\begin{gathered}
\left(-\frac{\hbar^{2}}{2 m^{*}} \nabla_{R}^{2}+\frac{1}{2} m^{*} \omega_{0}^{2} R^{2}+\frac{1}{2} \omega_{c} \widehat{L}_{R, Z}\right) \Psi_{N L}=E_{N L} \Psi_{N L} \\
\left(-\frac{\hbar^{2}}{2 m^{*}} \nabla_{r}^{2}+2 V_{0}+\frac{1}{2} m^{*} \Omega_{0}^{2} r^{2}+\frac{1}{2} \omega_{c} \widehat{L}_{r, z}\right) \Psi_{n l}=E_{n l} \Psi_{n l} \\
\left(\frac{1}{2} \omega_{c} g^{*} \hat{S}_{t o t, z}\right) \chi(\sigma)=E_{\text {spin }} \chi(\sigma)
\end{gathered}
$$

where $\Omega_{0}^{2}=\omega^{2}+\frac{\omega_{c}^{2}}{4}-2 \Omega^{2}$ and $\chi(\sigma)$ is the eigenstates of the spin operator $\hat{S}_{t o t, z}$. Eq. (4), Eq. (5) are a harmonic oscillator type with well-known eigenvalues and Eq. (6) is exactly soluble, so their eigenenergies are given by 


$$
\begin{gathered}
E_{R}=E_{N L}=\hbar \omega_{0}(2 N+|L|+1)+\frac{1}{2} L \hbar \omega_{c} \\
E_{r}=E_{n l}=2 V_{0}+\hbar \Omega_{0}(2 n+|l|+1)+\frac{1}{2} l \hbar \omega_{c} \\
E_{\text {spin }}=\frac{1}{2} \hbar \omega_{c} g^{*} \frac{\left[1-(-1)^{l}\right]}{2}=\frac{1}{2} \hbar \omega_{c} g^{*} S_{t o t, z}
\end{gathered}
$$

where $N, n=0,1,2, \ldots$ are the radial quantum numbers and $L, l=0 \pm 1, \pm 2, \ldots$ are the azimuthal quantum numbers, and the even and odd $l$ corresponds to the spin singlet $\left(S_{t o t, z}=0\right)$ and triplet $\left(S_{t o t, z}=1\right)$ states respectively.

We may notice that the energy levels in Eq. (4) are the well-known Fock-Darwin states (Chakraborty, 1999; Fock, 1928; Darwin, 1930) with eigenfunction given by

$$
\Psi_{N L}\left(R, \theta_{c m}\right)=\frac{1}{\sqrt{\pi}}\left(\sqrt{\frac{m^{*} \omega_{0}}{\hbar}}\right)^{1+|L|} \sqrt{\frac{N !}{(N+|L|) !}}(R)^{|L|} L_{N}^{|L|}\left(\frac{m^{*} \omega_{0}}{\hbar} R^{2}\right) e^{-m^{*} \omega_{0} R^{2} / 2 \hbar} e^{i L \theta_{c m}}
$$

where $L_{N}^{|L|}$ is the associated Laguerre polynomial. The complete energy spectrum for the parabolic quantum dot can be obtained analytically as

$$
E_{N, L, n, l, \text { spin }}=\hbar \omega_{0}(2 N+|L|+1)+\frac{1}{2} L \hbar \omega_{c}+2 V_{0}+\hbar \Omega_{0}(2 n+|l|+1)+\frac{1}{2} l \hbar \omega_{c}+\frac{1}{2} \hbar \omega_{c} g^{*} \frac{\left[1-(-1)^{l}\right]}{2}(11)
$$

Now, we will employ the canonical approach on the total energy spectrum to evaluate thermal and magnetic properties of the QD. Canonical formalism describes a fixed installation system that is in a state of thermal equilibrium with a heat bath for specific temperatures. The canonical ensemble contains varying states of energy but a similar structure; the different states in the ensemble given different probabilities depending on their total energy. The partition function can be calculated exactly and is given by (Greiner, Neise, \& Stocker, 1995; Landau \& Lifshitz, 1975)

$$
Z=\sum_{n=0}^{\infty} \sum_{l=-\infty}^{\infty} \sum_{N=0}^{\infty} \sum_{L=-\infty}^{\infty} \sum_{S_{t o t, z}=0}^{1} e^{-\frac{E_{N, L, n, l, S} S_{t o t, z}}{k_{B} T}}
$$

simplify, we get

$$
Z=\frac{e^{-\frac{2 V_{0}}{k_{B} T} \times e^{-\frac{\hbar \omega_{c} g^{*}}{4 k_{B} T}} \times \cosh \left(\frac{\hbar \omega_{c} g^{*}}{4 k_{B} T}\right)}}{\left[\cosh \left(\frac{\hbar \omega_{c}}{2 k_{B} T}\right)-\cosh \left(\frac{\hbar \omega_{0}}{k_{B} T}\right)\right]\left[\cosh \left(\frac{\hbar \omega_{c}}{2 k_{B} T}\right)-\cosh \left(\frac{\hbar \Omega_{0}}{k_{B} T}\right)\right]}
$$

Now, the mean internal energy for the system $\langle E\rangle=k_{B} T^{2}(\partial \ln Z / \partial T)$ is given by

$$
\begin{aligned}
& \langle E\rangle=2 V_{0}+\left[\cosh \left(\frac{\hbar \omega_{c}}{2 k_{B} T}\right)-\cosh \left(\frac{\hbar \omega_{0}}{k_{B} T}\right)\right]\left[\cosh \left(\frac{\hbar \omega_{c}}{2 k_{B} T}\right)-\cosh \left(\frac{\hbar \Omega_{0}}{k_{B} T}\right)\right] \times \operatorname{sech}\left(\frac{\hbar \omega_{c} g^{*}}{4 k_{B} T}\right) \cosh \left(\frac{\hbar \omega_{c} g^{*}}{4 k_{B} T}\right)
\end{aligned}
$$

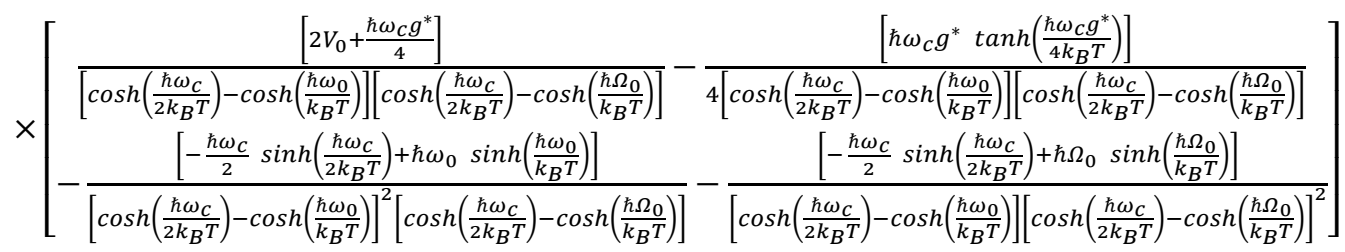

The heat capacity of a quantum dot is derived from the partition function as

$$
C_{V}=k_{B} T \frac{\partial^{2}(T \ln Z)}{\partial T^{2}}
$$

Also, Free energy presents valuable information on how likely the system transforms from one state to another, spontaneously. The less the free energy is the more stable the system. The Helmholtz free energy $F=-k_{B} T(\ln Z)$ comes out to be 


$$
\begin{aligned}
F= & k_{B} T \ln \left[\frac{\hbar \omega_{c} g^{*}}{4}\right]+2 V_{0}+k_{B} T \ln \left[\cosh \left(\frac{\hbar \omega_{c}}{2 k_{B} T}\right)-\cosh \left(\frac{\hbar \omega_{0}}{k_{B} T}\right)\right] \\
& +\left[\cosh \left(\frac{\hbar \omega_{c}}{2 k_{B} T}\right)-\cosh \left(\frac{\hbar \Omega_{0}}{k_{B} T}\right)\right]-k_{B} T \ln \left[\cosh \left(\frac{\hbar \omega_{c} g^{*}}{4 k_{B} T}\right)\right]
\end{aligned}
$$

The Helmholtz free energy is employed to obtain the entropy $\mathrm{S}=-\partial \mathrm{F} / \partial \mathrm{T}$ as

$$
S=k_{B} \log (Z)+\frac{1}{T}\langle E\rangle
$$

Also, mean energy was employed to calculate magnetization (M) and magnetic susceptibility $(\chi)$ as

$$
M=-\frac{\partial\langle E\rangle}{\partial B} \text { and } \chi=\frac{\partial M}{\partial B}
$$

It has been shown that in the regime of very large confinement potential the Harmonic interaction model has a good agreement with the full Coulomb problem; clearly the Harmonic interaction model is not valid for all electron separations $r=\left|\overrightarrow{r_{2}}-\overrightarrow{r_{1}}\right|$ (Johnson \& Kirczenow, 1993). In order to make the Harmonic interaction model more accurate in obtaining the results of the real interaction system, we could adjust the interaction parameters to produce the best fit for the real interaction of the dominant range of $r$. Equation (2) suggests that, for repulsion interaction $V\left(\vec{r}_{1}, \vec{r}_{2}\right)>0$ only if $r<r_{c}$, where $r_{c}=2 \sqrt{V_{0} / m^{*} \Omega^{2}}$. For physically true interaction, we should have $\bar{r}<r_{c}$, where $\bar{r}$ is the e-e separation implied by the wave function of the system as shown in ref. (Johnson, 1992) and it is given by $\bar{r}=\sqrt{\hbar / m^{*} \Omega_{0}}$. Thus, the critical e-e separation are obtained when $r_{c}=\bar{r}$, then we get

$$
\Omega=\frac{4 V_{0}}{\hbar} \sqrt{\left[\sqrt{1+\left(\frac{\hbar \omega}{4 V_{0}}\right)^{2}}-1\right]}
$$

By choosing effective mass of electron $\mathrm{m}^{*}=0.067 \mathrm{~m}_{\mathrm{e}}$ of a GaAs quantum dot, $\hbar \omega=2 \mathrm{meV}$ and $\mathrm{V}_{0}=10 \mathrm{meV}$, we calculate the critical interaction strength value $\Omega_{\mathrm{c}}\left(\Omega_{\mathrm{c}}=2.146 \times 10^{12} \mathrm{~Hz}\right)$, and this gives an acceptable fit to the true e-e interaction, where $r_{c}=1509.64 \AA$.

In this study, it's worth to mention that our study is performed in regime of large confinement potential where the Harmonic model can be used as a good approximation to Coulomb problem. Moreover, the main goal of this study is to show qualitatively the importance of taking the spin contribution into the calculation account especially when we study the magnetic properties of the quantum dot, since many previous studies have ignored the spin effect on these properties for two electrons quantum dot system.

In the coming section, the dependence of these properties for GaAs QD as a function of temperature (T), magnetic field (B) and spin are analyzed.

\section{Numerical Results and Discussion}

The theory in our study was applied to GaAs QD with $\mathrm{V}_{0}=10 \mathrm{meV}, \hbar \omega=2 \mathrm{meV}, \mathrm{m}^{*}=0.067 \mathrm{~m}_{\mathrm{e}}$ and $\hbar \Omega=$ $1 \mathrm{meV}$. Figure 1 shows the variation of the mean energy of (2DPQD) versus the temperature for different values of magnetic field also the spinless case is taking into account for comparison purposes. It is shown that the mean energy is enhanced as the temperature increases. However for higher temperatures a linear dependence on temperature is observed. This demonstrates a peak in the heat capacity, which in turn reminds us of entering the system in a mixed gas-liquid state. Also, the increase of magnetic field strength causes the increase in mean energy where the spin effect becomes significant. This is connected to the magnetic confinement effect that appears in the system strongly. The variation of the mean energy with the magnetic field for various values of temperature is shown in Figure 2. It is observed that the dependence of mean energy on magnetic field strength has a monotonic behavior. The figure shows that, at low values of magnetic field, mean energy increases monotonically with increasing magnetic field while at high values of magnetic field, this increase is almost linear. Also, it is noted for a fixed value of magnetic field, the mean energy decreases as the temperature decreases. The graph shows an important enhancement of the mean energy when the e-e Harmonic energy term is activated. 
Furthermore, when the magnetic field increase, the electrons are strongly confined in the QD, consequently the repulsive e-e Harmonic energy increases, and in effect the energy states, i.e., as the magnetic field increases, the magnetic confinement effects become more and more appreciable. It is noted that the spin effect is only noticeable in the high magnetic field values where it decreases the mean energy slightly.

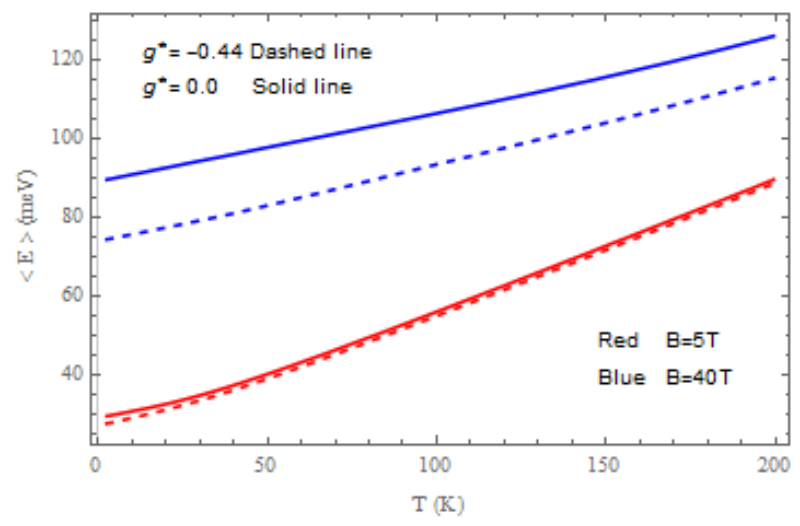

Figure 1. The average thermal energy $\langle E\rangle$ vs. temperature $(\mathrm{T})$ of $2 \mathrm{D}$ parabolic GaAs quantum dot for $\mathrm{B}=5$ Tesla and $\mathrm{B}=40$ Tesla

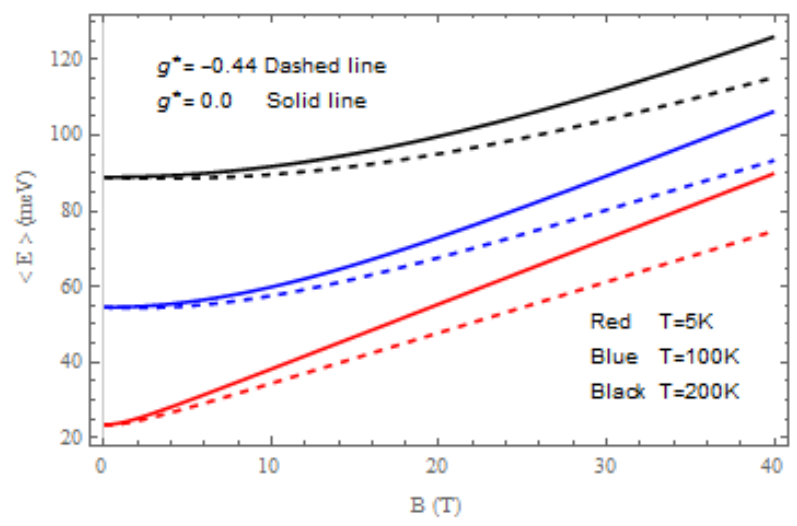

Figure 2. The average thermal energy $\langle E\rangle$ vs. the magnetic field (B) of 2D parabolic GaAs quantum dot for $\mathrm{T}=5$, 100 and $200 \mathrm{~K}$

In Figure 3, we have shown the dependence of thermal heat capacity upon temperature incorporating the effect of the electrons spin. We noticed that, heat capacity increases almost linearly at sufficiently low temperatures and a peak structure is observed due to the spin of the electrons. Also the peak shifts to the right as magnetic field is increased where its width becomes broader. Simply, this peak structure is attributed to Schottky anomaly for the heat capacity where just two states are of significance at low temperature since thermal energy picked up by electrons is sufficient for just the most minimal two levels. As the temperature rises, we find that, heat capacity reaches a saturation limit of $4 \mathrm{k}_{\mathrm{B}}$ in conformity with results reported by A. Chatterjee. J and M. M. Al Shorman (Boyacioglu \& Chatterjee, 2012; Al Shorman, Nammas, Haddad, \& Shukri, n. d.). The system exhibits a different behavior when subjected to a high and low magnetic field. For high magnetic field like $\mathrm{B}=15 \mathrm{~T}$, heat capacity increases steeply from 0 to $2 \mathrm{k}_{\mathrm{B}}$ and develops a shoulder and for small range of temperature it remains constant and with further increase in temperature heat capacity again starts to rise. While for low magnetic field like $B=2 T$, heat capacity increases sharply and reach the saturation limit more quickly with value of $4 \mathrm{k}_{\mathrm{B}}$ within a small temperature window. Additionally, to see the effect of spin on the heat capacity we have plotted the spin case and the spinless case in the same figure. It is clear that the significant effect of the spin appears in heat capacity at sufficiently high magnetic fields (results shown for $\mathrm{B}=15 \mathrm{~T}, 10 \mathrm{~T}$ ) and at low temperatures. The inclusion of spin in Hamiltonian gives an amazing behavior in the thermal and magnetic properties of the QD. The variation of heat capacity with magnetic field for distinct values of temperature is shown in Figure 4. For comparison purposes the spinless case is also plotted $\left(\mathrm{g}^{*}=0\right)$. We observed that, heat capacity exhibits a peak structure at very low values of magnetic field. These peaks related to a Schottky-like anomaly which is absent in 
the spinless case. Also the figure indicates that for high values of magnetic field, heat capacity curves merges and it reaches a constant value of $2 \mathrm{k}_{\mathrm{B}}$ and becomes independent of spin and temperature. Furthermore, at all temperatures, the hat capacity sharply decreases at very small $\mathrm{B}$ values except that for $\mathrm{T}=5 \mathrm{~K}$ where it increases.

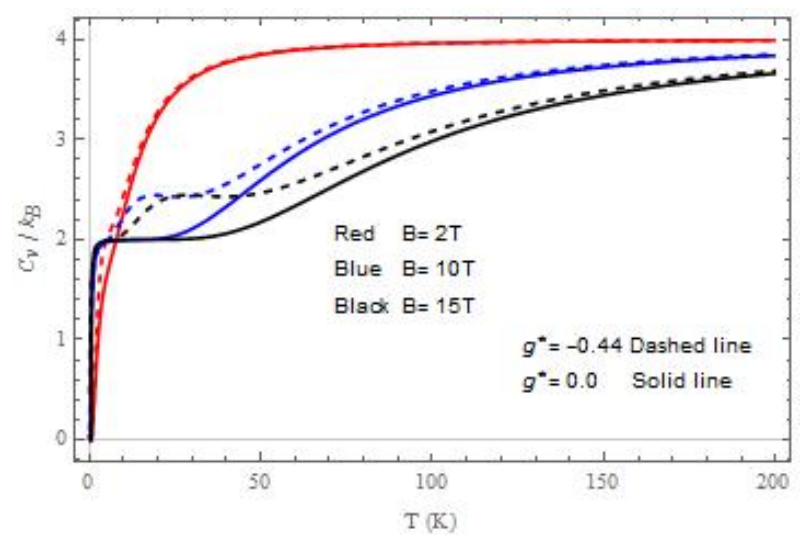

Figure 3. Heat capacity $\left(\mathrm{C}_{\mathrm{V}} / \mathrm{k}_{\mathrm{B}}\right)$ vs. temperature $(\mathrm{T})$ of $2 \mathrm{D}$ parabolic GaAs QD for $\mathrm{B}=2,10$ and 15 Tesla

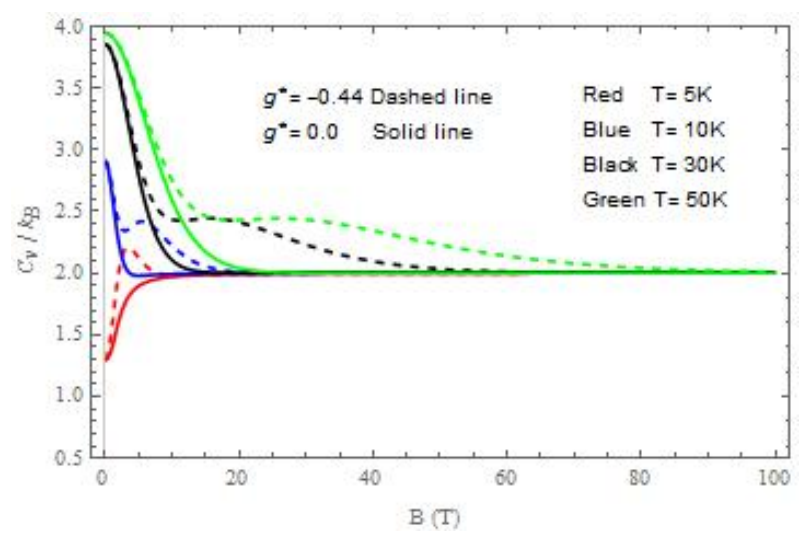

Figure 4. Heat capacity $\left(\mathrm{C}_{\mathrm{V}} / \mathrm{k}_{\mathrm{B}}\right)$ vs. magnetic field (B) of $2 \mathrm{D}$ parabolic GaAs $\mathrm{QD}$ for $\mathrm{T}=5,10,30$ and $50 \mathrm{~K}$

In Figure 5, we display the dependence of the heat capacity of the QD on the effective Lande $\mathrm{g}$ factor $\left(\mathrm{g}^{*}\right)$ for $\mathrm{B}=$ $15 \mathrm{~T}$ and $\mathrm{T}=5,30$ and $50 \mathrm{~K}$. The first observation we make is that heat capacity exhibits a double-peak structure and it shows a symmetric behavior about $\mathrm{g}^{*}=0$ where a local minima is observed. Second, it increases by increasing the Lande $g$ factor $g^{*}$, after which reaches its maximum value and then begins to decrease by increasing the Lande $g$ factor $\mathrm{g}^{*}$ and finally at a specific value of $\mathrm{g}^{*}\left(\left|\mathrm{~g}^{*}\right| \sim 1\right)$, it saturates to the value of $2 \mathrm{k}_{\mathrm{B}}$ and becomes independent of $g^{*}$, while the saturation for $T=50 \mathrm{~K}$ takes place at much larger $g^{*}$ values. The saturation of heat capacity at large $g^{*}$ is attributed to the fact that within a strong magnetic field, the spin moment of electron is saturated and the contribution of the Zeeman term to the mean energy becomes independent of temperature, beyond a specific value of $\mathrm{g}^{*}$ and as a result, there is no effect of $\mathrm{g}^{*}$ on heat capacity.

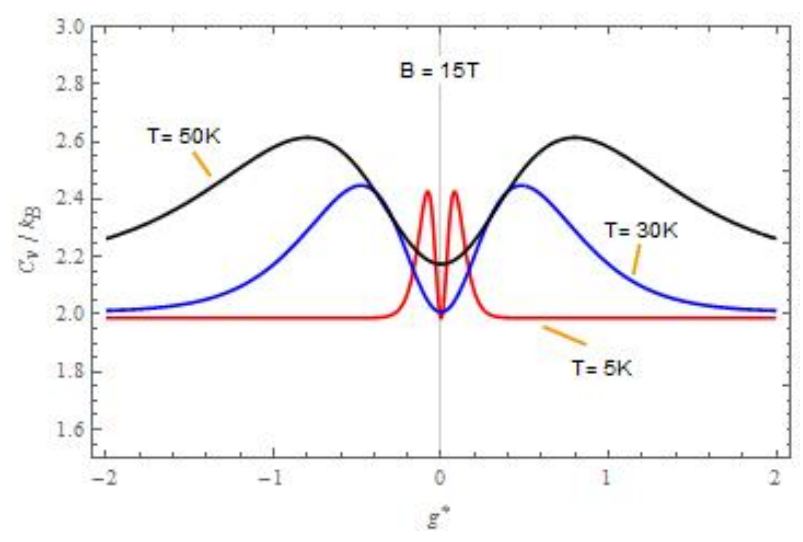

Figure 5. Heat capacity $\left(\mathrm{C}_{\mathrm{V}} / \mathrm{k}_{\mathrm{B}}\right)$ vs. $\mathrm{g}$ * of $2 \mathrm{D}$ parabolic GaAs $\mathrm{QD}$ for $\mathrm{T}=5,30$ and $50 \mathrm{~K}$ at $\mathrm{B}=15 \mathrm{Tesla}$ 
Figure 6 shows the variation of the entropy with temperature for various values of magnetic field. As we expect, entropy increases monotonically as the temperature increases. The increase in entropy with temperature is caused by the thermal enhancement of electrons which are working to bring more and more disorder in a random movement. It is noted that, entropy increases sharply at low temperatures where the effect of magnetic field becomes more and more pronounced, while in the high temperature region, entropy saturates. It should be noted that the spin does not show much change in the entropy, also at a fixed temperature, entropy increases by increasing the magnetic field. It is clear that the degree of random order of the system is confirmed by the interplay between the magnetic field and the temperature.

The dependence of entropy upon the magnetic field is shown in Figure 7 for different values of temperatures. It is seen that, at sufficiently low temperature like $\mathrm{T}=5 \mathrm{~K}$, entropy enhances as the magnetic field increases. But at relatively higher temperature like $\mathrm{T}=100 \mathrm{~K}$, entropy does not show a significant change in its value and becomes independent of both spin and magnetic field. However the spin does not affect the entropy significantly. This behavior can be mainly explained as follows, order brought into the system by magnetic field, can be balanced by kinetic energy due to confinement with the disturbance of thermal dynamics at higher temperatures.

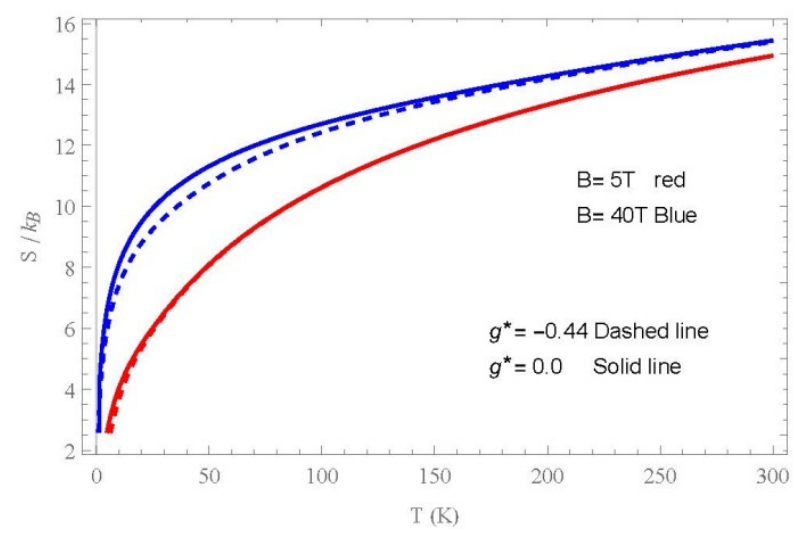

Figure 6. Entropy $\left(\mathrm{S} / \mathrm{k}_{\mathrm{B}}\right)$ vs. temperature $(\mathrm{T})$ of $2 \mathrm{D}$ parabolic GaAs $\mathrm{QD}$ for $\mathrm{B}=5$ Tesla and $\mathrm{B}=40$ Tesla

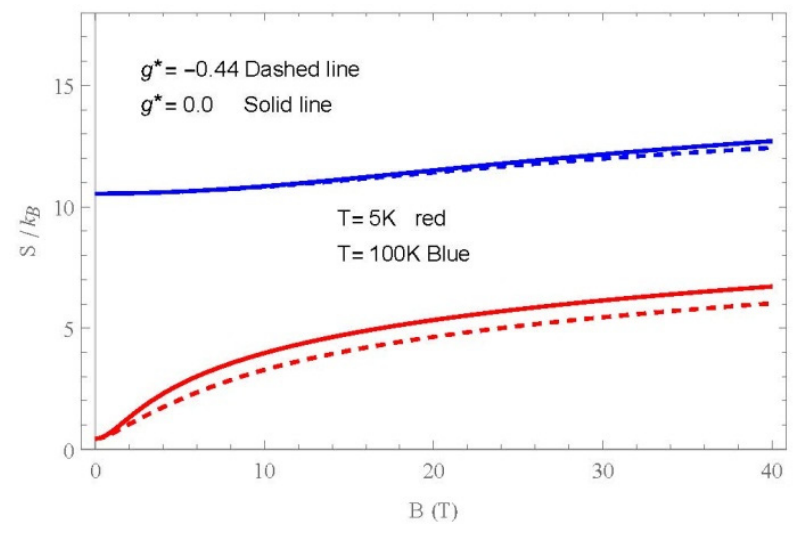

Figure 7. Entropy ( $\mathrm{S} / \mathrm{k}_{\mathrm{B}}$ ) vs. magnetic field (B) of $2 \mathrm{D}$ parabolic GaAs $\mathrm{QD}$ for $\mathrm{T}=5 \mathrm{~K}$ and $\mathrm{T}=100 \mathrm{~K}$

The magnetization as a function of temperature is shown in Figure 8. For comparison purposes, we have computed the magnetization with and without spin for different values of the magnetic field. One can observe that the magnetization is almost constant at sufficiently low temperatures up to a certain temperature (which depends on the magnetic field), the magnetization $|\mathrm{M}|$ decreases with increasing temperature at least, for the magnetic fields considered in this work. The decrease is faster whenever the magnetic field is larger. It is important to note that the characteristic behavior for GaAs quantum dots of diamagnetic materials fits the approach followed in our study (Ghaltaghchyan et al., 2016). It should be noted that at a fixed temperature the spin effect becomes more pronounced as the magnetic field increases.

In Figure 9, we plot the magnetization as a function of the applied magnetic field. We have shown the behavior of magnetization for four values of temperature with and without spin. We note from the figure that the magnetization decreases with increasing magnetic field and it is diamagnetic this is clear from the curves 
signature. We also observe that magnetization saturates to a constant for both $\mathrm{g}^{*}=0$ and $\mathrm{g}^{*}=-0.44$ within a small range of magnetic field since the system becomes fully polarized and becomes independent of temperature. It can also be seen that the effect of the spin is significantly increased by increasing the magnetic field strength; moreover the spin causes an increase in the magnetization in comparison with the spinless case.

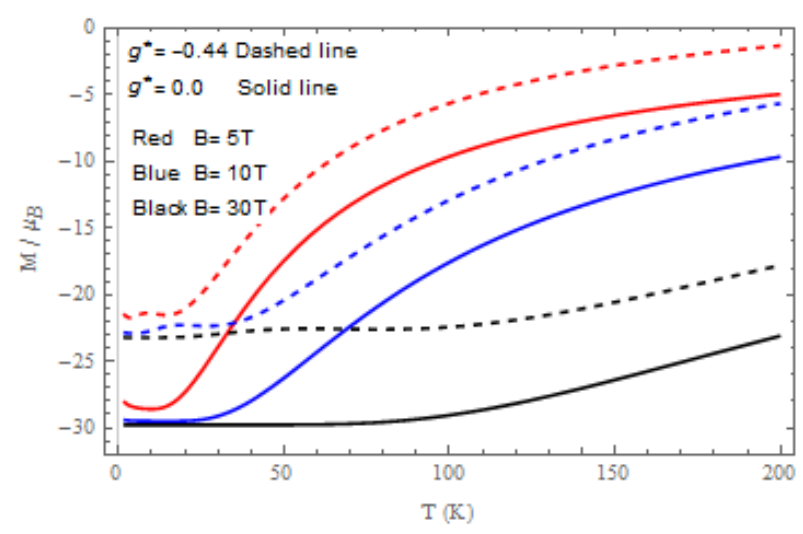

Figure 8. Magnetization $\left(M / \mu_{B}\right)$ vs. temperature $(\mathrm{T})$ of $2 \mathrm{D}$ parabolic GaAs QD for $\mathrm{B}=5,10$ and 30 Tesla

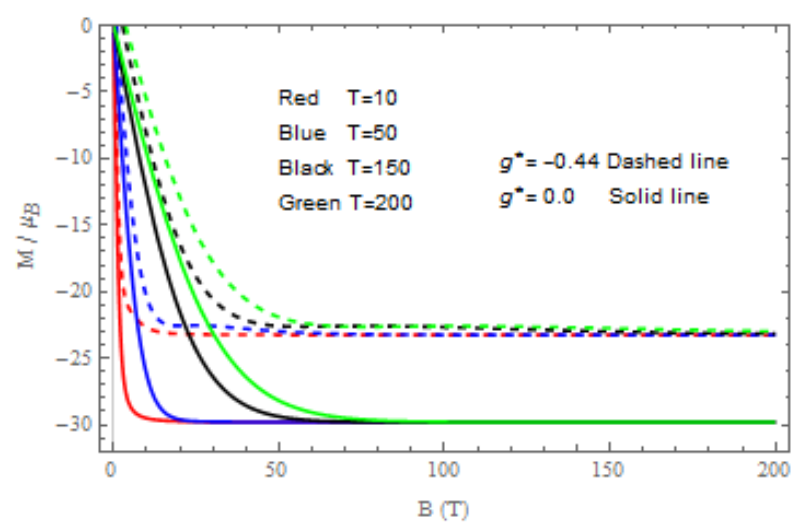

Figure 9. Magnetization $\left(M / \mu_{B}\right)$ vs. magnetic field (B) of 2D parabolic GaAs QD for T=10, 50, 150 and $200 \mathrm{~K}$

Variation of susceptibility with temperature is given in Figure 10 for different magnetic fields. For comparison purposes the spinless case is also plotted. The figure shows that the susceptibility initially decreases with temperature, because thermal agitation works to cause fluctuations in the polarized state of the system, then it reaches a minimum and then starts increasing with increasing temperature and finally at very large temperature the susceptibility attains a negative value and becomes independent of magnetic field and remains in a diamagnetic state. It can be noticed that the spin increases the susceptibility for all the values of temperature and magnetic field considered in this work. The reduction is more rapid for a lower magnetic field. The explicit magnetic field dependence of the susceptibility of the system at different temperatures with and without spin is shown in Figure 11. It is noted that, at lower temperatures like $\mathrm{T}=5 \mathrm{~K}, 25$ and $10 \mathrm{~K}$, the susceptibility enhances as the magnetic field increased, while at higher temperatures like $\mathrm{T}=50 \mathrm{~K}$, the susceptibility turns out to be independent of magnetic field at low magnetic field, at least, for the temperatures we have considered in this study. However, above a certain magnetic field value, the susceptibility increases with the increase in magnetic field. Finally, at very large magnetic field, the system is fully polarized, as a result, the overall susceptibility reduces to zero and becomes independent of temperatures. This is in conformity with magnetization behavior shown in Figure 9. One can observe that the spin does not show a significant effect on the susceptibility also the susceptibility is diamagnetic for the present system. 


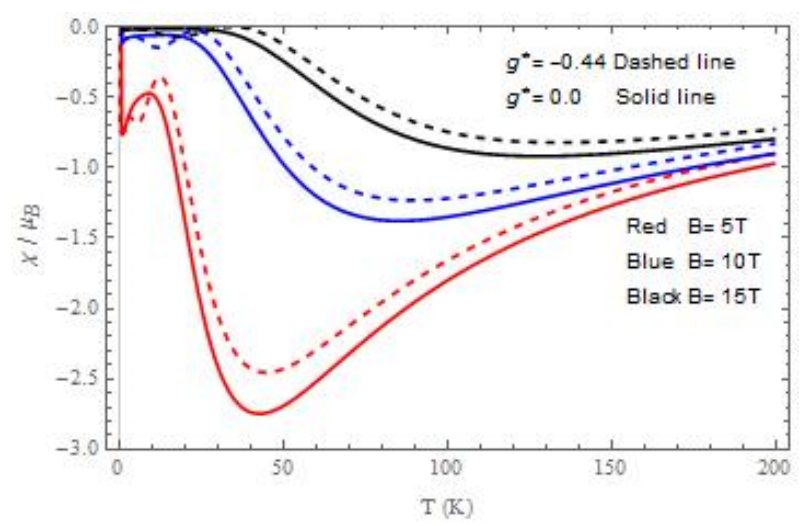

Figure 10. Susceptibility $\left(\chi / \mu_{B}\right)$ vs. temperature (T) of 2D parabolic GaAs QD for B=5, 10 and 15 Tesla

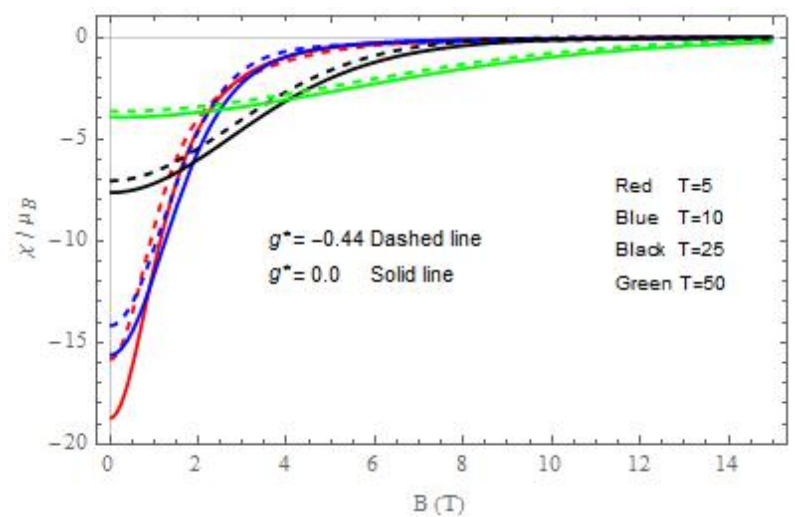

Figure 11. Susceptibility $\left(\chi / \mu_{B}\right)$ vs. B of $2 \mathrm{D}$ parabolic GaAs QD for $\mathrm{T}=5,10,25$ and $50 \mathrm{~K}$

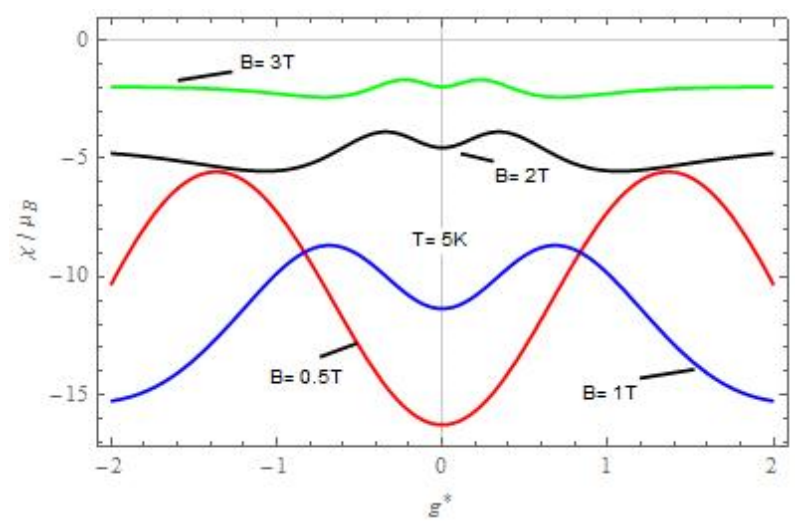

Figure 12. Susceptibility $\left(\chi / \mu_{B}\right)$ vs.g of $2 \mathrm{D}$ parabolic GaAs $\mathrm{QD}$ for $\mathrm{B}=0.5,1,2$ and 3 Tesla at $\mathrm{T}=5 \mathrm{~K}$

In Figure 12 we display the dependence of the susceptibility upon the Lande $g$ factor $\left(\mathrm{g}^{*}\right)$ for four values of magnetic field strength at $\mathrm{T}=5 \mathrm{~K}$. We note that the susceptibility shows a symmetric behavior about $\mathrm{g}^{*}=0$. This can be comprehended from the susceptibility equation which can be acquired from Eq. (18). It is very simple to see that at finite values of magnetic field and temperature $\hbar \omega_{c} g^{*} \ll k_{B} T$, and therefore susceptibility is proportional to the square of the Lande $\mathrm{g}$ factor $\left(\mathrm{g}^{*}\right)$. In addition, a local minimum is observed in susceptibility at $\mathrm{g}^{*}=0$ and susceptibility is completely diamagnetic. This is too effortlessly reasonable. For $\mathrm{g}^{*}=0$ the magnetic susceptibility is in a diamagnetic state. Also, the maximum is noticeable at a lower values of $\mathrm{g}^{*}$ for the lower magnetic field (results shown for $\mathrm{B}=0.5 \mathrm{~T}$ and $\mathrm{B}=1 \mathrm{~T}$ ). But, as $\mathrm{g}^{*}$ increases, at a fixed magnetic field, susceptibility begins increasing and this justifies the minimum at $\mathrm{g}^{*}=0$. However, as $\mathrm{g}^{*}$ increases diamagnetic effect increases until magnetization saturates. So, one would expect a maximum in susceptibility at this value of $\left|\mathrm{g}^{*}\right|$. Beyond this value of $\mathrm{g}^{*}$, diamagnetism decreases rapidly. While, for $\mathrm{B}=2 \mathrm{~T}$ and $3 \mathrm{~T}$ we notice that the 
susceptibility increases as $\mathrm{g}^{*}$ increases, and then with further increase in $\mathrm{g}^{*}$ the susceptibility decreases smoothly till susceptibility becomes independent of $\mathrm{g}^{*}$. Also, a double-peak structure is observed and it becomes noticeable as the magnetic field decreases (results shown for $B=0.5 T$ and $B=1 T$ ). This study of susceptibility as a function of Lande $\mathrm{g}$ factor can be utilized to find the correct materials parameters to achieve certain magnetic properties.

\section{Conclusion}

The thermal and magnetic properties of a two Harmonically interacting electrons in a GaAs quantum dot with parabolic confinement are investigated as a function of temperature and magnetic field taking into consideration the spin-Zeeman energy. We obtained the energy spectrum of the system in closed form by solving Schrodinger equation analytically and then we find heat capacity, entropy, magnetization and susceptibility using the canonical ensemble approach. As a function of temperature, it was demonstrated that the heat capacity exhibits a peak structure at a very low temperature, while at high temperature it saturates to the value of $4 \mathrm{k}_{\mathrm{B}}$ and it was noted that the spin increases heat capacity only at low temperature and at high magnetic field. We also found that, at low temperature entropy increases steeply as the temperature increases, and at very high temperatures the entropy reaches the saturation limit. It was found that the spin does not affect the entropy significantly. Also it was shown that, at large magnetic field like $B=30 \mathrm{~T}$, the magnetization remains constant with a temperature up to a certain value, beyond which the magnetization begins to increase. As it turns out that the spin leads to an increase in magnetization. It is found that, at very large temperatures the susceptibility saturates to a negative value and becomes independent of magnetic field. However, it was noted that the diamagnetic state is the preferred state of our system. Also, it was noted that the spin always increases the susceptibility irrespective the value of magnetic field and temperature. As a function of magnetic field, the heat capacity shows at very low values of magnetic field a Schottky-like anomaly which is absent in the spinless case, but with further increase in magnetic field, heat capacity becomes independent of the spin and temperature and attains a constant value of $2 \mathrm{k}_{\mathrm{B}}$. It was also shown that, for sufficiently low temperatures like $\mathrm{T}=5 \mathrm{~K}$, entropy enhances with increasing in magnetic field. While at $\mathrm{T}=100 \mathrm{~K}$, entropy is almost constant and does not show a significant change in its value and it shows very little dependence on both the magnetic field and the spin. It was observed that the magnetization decreases sharply with increasing magnetic field and it is diamagnetic and saturates to a constant as the magnetic field increases. Moreover, the inclusion of the spin on the magnetization is tangible as the magnetic field increases. The susceptibility was found to increase with increasing magnetic field and then reaches zero at high values of the magnetic field, also the spin does not show a significant effect on the susceptibility. As a function of the Lande $g$ factor, the heat capacity exhibits a local minima at $g^{*}=0$, and it saturates at a certain value of $g^{*}$ with value of $2 \mathrm{k}_{\mathrm{B}}$, while the susceptibility shows a double-peak structure at very low magnetic field, but at comparatively large magnetic field, susceptibility becomes almost independent of $\mathrm{g}^{*}$ with further increase in $\mathrm{g}^{*}$. It was concluded that, the main effect of the spin is an increase in the magnitude of these properties; moreover in the absence of spin, there is no change in the general behavior of these properties. The possibility of controlling the properties of these magnetic materials would be significantly charming in the coming technology from key points of view, especially in context of the continuous headways in magnetodevices and spintronics.

In future work, it is planned to investigate these properties of two Harmonically interacting electrons confined in two-dimensional Gaussian quantum dot in the presence of both magnetic field and spin-Zeeman energy using the canonical ensemble approach and study their behavior on temperature, magnetic field, QD dot size and other relevant parameters.

\section{Conflict of interests}

The authors declare that there is no conflict of interests regarding the publication of this paper.

\section{References}

Al Shorman, M. M., Nammas, F. S., Haddad, H., \& Shukri, A. A. (n. d.). Chinese Journal of Physics, 56(3), 1057-1063.

Ashoori, R. C. (1996). Nature, 379, 413.

Beenakker, C. W. J. (1991). Phys. Rev. B, 44, 1646.

Bimberg, D., Grundmann, M., \& Ledentsov, N. N. (1998). Quantum Dot Heterostructures. Wiley, New York.

Boda, A., \& Chatterjee, A. (1942). AIP Conf. Proc., 050017-1-050017-4

Boda, A., \& Chatterjee, A. (2014). Physica B, 448, 244-246.

Boda, A., Kumar, D. S., Sankar, I. V., \& Chatterjee, A. (2016). J. Mag. Mag. Mater., 418, 242. 
Boyacioglu, B., \& Chatterjee, A. (2012). J. Appl. Phys., 112, 083514.

Chakraborty, T. (1999). Quantum Dots: A Survey of the Properties of the Artificia Atoms (1st ed.). Elsevier Science, Netherlands.

Chang, C. Y., \& Sze, S. M. (1996). ULSI Technology. McGraw-Hill, New York.

Cipriani, G., Rosa-Clot, M., \& Taddei, S. (2000). Phys. Rev. B, 61, 7536.

Climente, J. I., Planelles, J., \& Movilla, J. L. (2004). Physical Review B, 70, 081301(R).

Darwin, C. G. (1930). Mathematical Proc. Cambridge Philos. Soc., 27, 86.

De Groote, J., Horonos, J. E. M., \& Chaplik, A. V. (1992). Phys. Rev. B, 46(19), 12773.

Emperador, A., Lipparini, E., \& Serra, L. (2006). Phys. Rev. B, 73, 235341.

Ferconi, M., \& Vignale, G. (1994). Phys. Rev. B, 50, 14722.

Fock, V. (1928). Z. Phys., 47,446.

Ghaltaghchyan et al. (2016). J. Contemp. Phys., 51, 162.

Greiner, W., Neise, L., \& Stocker, H. (1995). Thermodynamics and Statistical Mechanics. Springer, New York.

Gu, S. W., \& Guo, K. X. (1993). Solid State Commun, 89, 1023.

Haddad, H., Nammas, F. S., Al Shorman, M. M., \& Shukri, A. A. (2017). Physica B: Condenced matter, 526, 132-135.

Harju, A., Sverdlov, V. A., Barbiellini, B., \& Nieminen, R. M. (1998). Physica B, 255, 145.

Harju, A., Sverdlov, V. A., Nieminen, R. M., \& Halonen, V. (1999). Phys. Rev. B, 59, 5622.

Hawrylak, P. (1993). Phys. Rev. Lett., 71, 3347.

Jacak, L., Hawrylack, P., \& Wojs, A. (1998). Quantum Dot. Springer, Berlin.

Jackson, J. D. (1962). Classical Electrodynamics. John Wiley \& 22 Sons, New York.

Johnson, B. L., \& Kirczenow, G. (1993). Phys. Rev. B, (47), 10563.

Johnson, N. F. (1992). J. Phys.: Condens. Matter, 4(42), 555-562.

Johnson, N. F. (1995). Journal of Physics: Condensed Matter, 7, 965.

Johnson, N. F., \& Payne, M. C. (1991). Phys. Rev. Lett., 67, 1157.

Johnson, N. F., \& Payne, M. C. (1992). Phys. Rev. B, 45, 3819

Johnson, N. F., \& Reina, M. (1992). J. Phys.: Condens. Matter, 4, L623.

Karrai, K., Drew, H. D., Lee, M. W., \& Shayengan, M. (1989). Phys. Rev. B, 39, 1426.

Kastner, M. A. (1992). Reviews of Modern Physics, 64, 849.

Kouwenhoven, L. P., Austing, D. G., \& Tarucha, S. (2001). Reports on Progress in Physics, 64, 701.

Kuros, A., \& Okopinska, A. (2015). Few-Body Syst., 56, 853.

Landau, L. D., \& Lifshitz, E. M. (1975). Statistical Physics (3rd ed.). Pergamon International Library. New York.

Li, Q. P., Karrai, K., Yip, S. K., Sarma, S. D., \& Drew, H. D. (1991). Phys. Rev. B, 43, 5151.

Liu et al. (2017). Journal of Physical Chemistry C, 121, 26086-26095.

Liu, J. L. (2012). Mathematical modeling of semiconductor quantum dots based on the nonparabolic effective-mass approximation. Nano MMTA, 1, 58.

Maksym, P. A., \& Chakraborty, T. (1990). Phys. Rev. Lett., 65, 108.

McEuen, P. L., Foxman, E. B., Kinaret, J., Meirav, U., Kastner, M. A., Wingreen, N. S., \& Wind, S. J. (1992). Phys. Rev. B, 45(R), 11419.

Merkt, U., Huser, J., \& Wagner, M. (1991). Phys. Rev. B, 43, 7320.

Mukhopadhyay, S., \& Chatterjee, A. (1996). Int. J. Mod. Phys., 10, 2781.

Mukhopadhyay, S., \& Chatterjee, A. (1997). Phys. Rev. B, 55, 9279.

Mukhopadhyay, S., \& Chatterjee, A. (1999a). J. Phys.: Condens. Matter, 11, 2071. 
Mukhopadhyay, S., \& Chatterjee, A. (1999b). Phys. Rev. B, 59, R7833.

Mukhopadhyay, S., \& Chatterjee, A. (2000). Int. J. Mod. Phys. B, 14, 3897.

Mukhopadhyay, S., \& Chatterjee, A. (2001). Acta Physica Polonica B, 32, 473.

Nammas, F. S. (2018). Physica A, 508, 187-198.

Nammas, F., Sandouqa, A., Ghassib, H., \& Al-Sugheir, M. (2011). Physica B, 406, 4671-4677.

Oaknin, J. H., Palacios, J. J., Brey, L., \& Tejedor, C. (1994). Phys. Rev. B, 49, 5718.

Peeters, F. M. (1990). Phys. Rev. B, 42, 1486.

Reusch, B., \& Grabert, H. (2003). Phys. Rev. B, 68, 045309.

Shaer, A., Elsaid, M. K., \& Elhasan, M. (2016). Chinese Journal of Physics, 54(3), 391-397.

Shaer, A., Elsaid, M. K., \& Elhasan, M. (n. d.). Turkish Journal of Physics, 40(3), 209-218.

Sharma et al. (2019). Journal of Magnetism and Magnetic Materials, 469, 171-177.

Sikorski, C., \& Merkt, U. (1989). Phys. Rev. Lett., 62, 2164.

Sze, S. M. (1981). Physics of Semiconductor Devices. John Wiley \& Sons, New York.

Sze, S. M. (1985). Semiconductor Devices Physics and Technology. John Wiley \& Sons, New York.

Tavernier, M. B., Anisimovas, E., Peeters, F. M., Szafran, B., Adamowski, J., \& Bednarek, S. (2003). Phys. Rev. $B, 68,205305$.

Woggon, U. (1997). Optical Properties of Semiconductor Quantum Dots. Springer,Berlin.

Yang, S. R. E., MacDonald, A. H., \& Johnson, M. D. (1993). Phys. Rev. Lett., 71, 3194.

Yip, S. K. (1991). Phys. Rev. B, 43, 1707.

\section{Copyrights}

Copyright for this article is retained by the author(s), with first publication rights granted to the journal.

This is an open-access article distributed under the terms and conditions of the Creative Commons Attribution license (http://creativecommons.org/licenses/by/4.0/). 